\title{
Método de Elementos Finitos de Galerkin Descontínuo para Equações de Navier-Stokes Bidimensionais
}

I. MOZOLEVSKI ${ }^{1}$, Departamento de Matemática, UFSC, 88040-900 Trindade, Florianópolis, SC, Brasil

P.R. BÖSING ${ }^{2}$, Departamento de Matemática Aplicada, IME, USP, Rua do Matão 1010, 05508-090 São Paulo, SP, Brasil.

\begin{abstract}
Resumo. Neste trabalho apresentamos um método de Galerkin descontínuo para as equações de Navier-Stokes incompressíveis, bidimensionais em regime permanente. Usando a formulação da função corrente, o problema se reduz para uma equação biharmônica não-linear que é linearizada com o método de iteração de Picard. Para a equação biharmônica linear, apresentamos uma formulação com penalização interior do método de elementos finitos de Galerkin descontínuo. Esta formulação é o resultado da combinação de outras duas formulações, uma para a parte elíptica e outra para parte hiperbólica do problema. São apresentados resultados numéricos que confirmam a eficiência do método na resolução numérica das equações de Navier-Stokes para uma ampla escala do número de Reynolds.
\end{abstract}

\section{Introdução}

A resolução numérica das equações de Navier-Stokes tem sido o objeto de estudo em dinâmica de fluidos computacionais há muitas décadas. Em particular, os avanços tecnológicos dos últimos anos contribuíram fortemente para o desenvolvimentos destas pesquisas. Em [8], pode-se encontrar uma revisão dos métodos numéricos mais comuns aplicáveis às equações de Navier-Stokes incompressíveis. Dentre estes métodos, os métodos de elementos finitos sempre tiveram grande destaque devido a sua adaptatividade, estabilidade e precisão. Para mais detalhes veja [7] e [10]. Em [2], [5] e [4] foi introduzido, analisado e numericamente testado, um método de elementos finitos de Galerkin descontínuo local para as equações de Stokes, equações de Oseen, e para as equações de Navier-Stokes incompressíveis, respectivamente (veja [3] para uma revisão). Nestes trabalhos, foi demonstrado que o método é um método misto estabilizado, localmente conservativo e tem alta ordem de precisão.

Neste trabalho, vamos considerar as equações de Navier-Stokes bidimensionais, incompressíveis e em regime permanente. Então, introduzindo uma função

\footnotetext{
${ }^{1}$ igor@mtm.ufsc.br - Apoio parcial do CNPq, Brasil

${ }^{2}$ paulo@ime.usp.br - Bolsista do CNPq, Brasil
} 
corrente, reduzimo-a a uma equação biharmônica não-linear, e tratamos essa não linearidade usando o método de iteração de Picard. Para essa equação resultante, vamos introduzir uma formulação com penalização interior de Galerkin descontínuo. Essa formulação é obtida combinando a apresentada em [11, 9] para a equação biharmônica, com a apresentada em [1] para equações hiperbólicas de primeira ordem. Os testes numéricos realizados comprovam que a estratégia usada é eficiente, pois os resultados obtidos condizem com os apresentados na literatura.

A estrutura deste trabalho é composta de quatro seções. Na seção 2, reduzimos as equações de Navier-Stokes á uma equação linear de quarta ordem, usando função corrente e iteração de Picard. A seção 3, é dedicada aos espaços de elementos finitos e a construção do método de Galerkin descontínuo. E por último, na seção 4, usamos o método apresentado para resolver numericamente o problema do fluxo na cavidade em 2D. Esse problema é usado geralmente como modelo para teste e avaliação de muitas técnicas numéricas para equações de Navier-Stokes.

\section{Formulação Matemática}

Consideramos as equações de Navier-Stokes bidimensionais, incompressíveis e em regime estacionário

$$
-\nu \triangle \mathbf{v}+\mathbf{v} \cdot \nabla \mathbf{v}+\nabla p=\mathbf{F} \quad \text { em } \Omega
$$

e a equação da continuidade

$$
\nabla \cdot \mathbf{v}=0 \quad \text { em } \Omega
$$

com a condição de contorno

$$
\mathbf{v}=\mathbf{g} \text { sobre } \partial \Omega \text {. }
$$

Nestas equações, v é o campo de velocidades, $p$ é a pressão, $\nu$ é a viscosidade cinemática do fluido e $\mathbf{F}$ é a força externa. Vamos assumir que $\Omega \in \Re^{2}$ é um domínio poligonal convexo limitado com fronteira $\partial \Omega$, e que a função $\mathbf{g}$ satisfaz a condição de compatibilidade $\int_{\partial \Omega} \mathbf{g} \cdot \mathbf{n} d s=0$, sendo $\mathbf{n}$ o vetor normal unitário a $\partial \Omega$ e exterior a $\Omega$.

Com o uso da função corrente $\psi$, definida por

$$
u=\frac{\partial \psi}{\partial y} ; \quad v=-\frac{\partial \psi}{\partial x},
$$

combinamos a equação de Navier-Stokes e a equação da continuidade e obtemos a seguinte equação biharmônica não-linear:

$$
\nu \triangle^{2} \psi-\frac{\partial}{\partial x}\left(\frac{\partial \psi}{\partial y} \triangle \psi\right)+\frac{\partial}{\partial y}\left(\frac{\partial \psi}{\partial x} \triangle \psi\right)=f \quad \text { em } \Omega .
$$

Na parte não-linear desta equação, empregamos o método de iteração de Picard. Assim, derivamos o seguinte problema de valor de fronteira:

$$
\nu \triangle^{2} \psi-\frac{\partial}{\partial x}\left(v_{1} \triangle \psi\right)-\frac{\partial}{\partial y}\left(v_{2} \triangle \psi\right)=f \quad \text { em } \Omega,
$$




$$
\begin{aligned}
& \psi=g_{0} \text { sobre } \partial \Omega, \\
& \mathbf{n} \cdot \nabla \psi=g_{1} \quad \text { sobre } \partial \Omega,
\end{aligned}
$$

em que $f \in L_{2}(\Omega), \quad v_{1}, v_{2} \in C^{(1)}(\bar{\Omega})$

Na próxima seção, vamos apresentar uma $h p$-versão do método de elementos finitos de Galerkin descontínuo (discontinuous Galerkin finite element method DGFEM) com penalização interior para o problema de valor de fronteira (2.1)(2.2). Para aproximar o operador biharmônico desta equação, vamos usar a formulação do DGFEM não-simétrico apresentado em [11, 9] e, para aproximar o termo hiperbólico, empregaremos o método de Galerkin descontínuo estabilizado, introduzido em [1].

\section{Formulação do DGFEM}

Seja $\left\{\mathcal{T}_{h}\right\}(h>0)$ uma família regular de partições de $\Omega$ composta por elementos $\kappa_{i}$, abertos, disjuntos e convexos de maneira que,

$$
\bar{\Omega}=\bigcup_{\kappa \in \mathcal{T}_{h}} \bar{\kappa} .
$$

Assumimos que cada elemento $\kappa \in \mathcal{T}_{h}$ é imagem de um elemento mestre fixo $\widehat{\kappa}$ por uma aplicação afim, isto é, $\kappa=\mathcal{F}_{\kappa}(\widehat{\kappa})$. O elemento mestre aqui considerado é o quadrado unitário aberto em $\Re^{2}$.

Sobre a partição $\mathcal{T}_{h}$, consideramos a função constante por partes $h_{\mathcal{T}_{h}}$ definida por

$$
h_{\mathcal{T}_{h}}(x)=h_{\kappa}=\operatorname{diam}(\kappa), \quad x \in \kappa, \quad \kappa \in \mathcal{T}_{h},
$$

e designamos por $h$ o máximo dos $h_{\kappa}, \quad \kappa \in \mathcal{T}_{h}$.

Para um $m$ inteiro positivo, $Q_{m}(\widehat{\kappa})$ denotará o espaço do produto tensorial dos polinômios de grau menor ou igual a $m$ em cada direção coordenada, definidos sobre o elemento mestre $\widehat{\kappa}$. Para $\kappa \in \mathcal{T}_{h}$, associamos o valor do grau de aproximação polinomial local $p_{\kappa}$ e coletando $p_{\kappa}$ e $\mathcal{F}_{\kappa}$ em vetores $\mathbf{p}=\left\{p_{\kappa}: \kappa \in \mathcal{T}_{h}\right\}$ e $\mathcal{F}=$ $\left\{\mathcal{F}_{\kappa}: \kappa \in \mathcal{T}_{h}\right\}$, podemos definir o seguinte espaço de elementos finitos:

$$
S^{\mathbf{p}}\left(\Omega, \mathcal{T}_{h}, \mathcal{F}\right)=\left\{v \in L^{2}(\Omega): v_{\mid \kappa} \circ \mathcal{F}_{\kappa} \in Q_{p_{\kappa}}(\widehat{\kappa}), \quad \forall \kappa \in \mathcal{T}_{h}\right\} .
$$

Seja $\mathcal{E}_{h}$ o conjunto de todas as faces abertas $e$, de todos os elementos $\kappa \in \mathcal{T}_{h}$. Sobre este conjunto consideramos a função constante por partes $h_{\mathcal{E}_{h}}$ definida por

$$
h_{\mathcal{E}_{h}}(x)=h_{e}=\operatorname{diam}(e), \quad x \in e, \quad e \in \mathcal{E}_{h} .
$$

Sendo a família $\left\{\mathcal{T}_{h}\right\}$ regular, existe uma constante positiva $c$, independente de $h$, tal que,

$$
c h_{\kappa} \leq h_{e} \leq h_{\kappa}, \quad \forall \kappa \in \bigcup_{h>0} \mathcal{T}_{h}, \quad \forall e \in \partial \kappa .
$$

O conjunto $\mathcal{E}_{h}$ será dividido em dois subconjuntos, $\mathcal{E}_{h}^{\circ}$ e $\mathcal{E}_{h}^{\partial}$, definidos por

$$
\begin{aligned}
\mathcal{E}_{h}^{\circ} & =\left\{e \in \mathcal{E}_{h}: e \subset \Omega\right\}, \\
\mathcal{E}_{h}^{\partial} & =\left\{e \in \mathcal{E}_{h}: e \subset \partial \Omega\right\} .
\end{aligned}
$$


Além disso, designamos

$$
\Gamma^{\circ}=\left\{x \in \Omega: x \in e \text { para } e \in \mathcal{E}_{h}^{\circ}\right\}
$$

e $\Gamma=\Gamma^{\circ} \cup \partial \Omega$.

Para qualquer face $e \in \mathcal{E}_{h}^{\circ}$ há dois elementos $\kappa_{i}$ e $\kappa_{j}(i>j)$, tais que, $\overline{\kappa_{i}} \cap \overline{\kappa_{j}}=\bar{e}$. Então, para qualquer $m$ inteiro,

$$
\left\{p^{m}\right\}_{\mathcal{E}_{h}}(x)=\left\{p^{m}\right\}_{e}= \begin{cases}\frac{p_{\kappa_{i}}^{m}+p_{\kappa_{j}}^{m}}{p_{\kappa}^{m},}, & \text { se } e \in \mathcal{E}_{h}^{\circ} \\ p_{\kappa}^{m}, e \in \mathcal{E}_{h}^{\partial}\end{cases}
$$

e, para qualquer função $v \in H^{s}\left(\Omega, \mathcal{T}_{h}\right), s>1 / 2$, definimos a média e o salto (dependem da enumeração dos elementos) de $v$ sobre $e$ por

$$
\begin{aligned}
\{v\} & = \begin{cases}\frac{1}{2}\left(v_{\mid \kappa_{i}}\right)_{\mid e}+\frac{1}{2}\left(v_{\mid \kappa_{j}}\right)_{\mid e}, & \text { se } e \in \mathcal{E}_{h}^{\circ} \\
\frac{1}{2} v_{\mid e}, & \text { se } e \in \mathcal{E}_{h}^{\partial}\end{cases} \\
{[v] } & = \begin{cases}\left(v_{\mid \kappa_{i}}\right)_{\mid e}-\left(v_{\mid \kappa_{j}}\right)_{\mid e}, & \text { se } e \in \mathcal{E}_{h}^{\circ} \\
v_{\mid e}, & \text { se } e \in \mathcal{E}_{h}^{\partial},\end{cases}
\end{aligned}
$$

respectivamente. Além disso, para cada face $e \in \mathcal{E}_{h}^{\circ}$, associamos o vetor normal unitário $\nu=\mathbf{n}_{\kappa_{i}}$ para $e$, que aponta de $\kappa_{i}$ para $\kappa_{j}$ e, para cada face $e \in \mathcal{E}_{h}^{\partial}$, associamos o vetor normal unitário exterior $\nu=\mathbf{n}_{\kappa}$, em que $e \subset \partial \kappa$.

Para $\psi, \phi \in L^{2}(\Gamma)$, definimos o seguinte produto interno

$$
\langle\psi, \phi\rangle_{L^{2}(\Gamma)}=\int_{\Gamma^{\circ}} \psi \phi \mathrm{d} s+\int_{\partial \Omega} \psi \phi \mathrm{d} s,
$$

ao qual associamos a norma $\|\cdot\|_{L^{2}(\Gamma)}$.

Introduzimos, agora, a seguinte forma bilinear associada à equação (2.1):

$$
\mathcal{B}_{\mathrm{DG}}(\psi, \phi)=\mathcal{B}_{e}(\psi, \phi)+\mathcal{B}_{a}(\psi, \phi),
$$

em que

$$
\begin{aligned}
\mathcal{B}_{e}(\psi, \phi) & =\sum_{\kappa \in \mathcal{T}_{h}}(\Delta \psi, \Delta \phi)_{L^{2}(\kappa)} \\
& +\langle\{\nu \cdot \nabla(\Delta \psi)\},[\phi]\rangle_{L^{2}(\Gamma)}+\lambda_{1}\langle\{\nu \cdot \nabla(\Delta \phi)\},[\psi]\rangle_{L^{2}(\Gamma)} \\
& -\left(\langle\{\Delta \psi\},[\nu \cdot \nabla \phi]\rangle_{L^{2}(\Gamma)}+\lambda_{2}\langle\{\Delta \phi\},[\nu \cdot \nabla \psi]\rangle_{L^{2}(\Gamma)}\right) \\
& +\langle\alpha[\psi],[\phi]\rangle_{L^{2}(\Gamma)}+\langle\beta[\nu \cdot \nabla \psi],[\nu \cdot \nabla \phi]\rangle_{L^{2}(\Gamma)}
\end{aligned}
$$

corresponde à parte elíptica do problema e

$$
\begin{aligned}
\mathcal{B}_{a}(\psi, \phi) & =\sum_{\kappa \in \mathcal{T}_{h}}(\Delta \psi,(\mathbf{U} \cdot \nabla \phi))_{L^{2}(\kappa)} \\
& -\langle(\mathbf{U} \cdot \nu)\{\Delta \psi\},[\phi]\rangle_{L^{2}(\Gamma)}-\langle\gamma[\Delta \psi],[\phi]\rangle_{L^{2}\left(\Gamma^{\circ}\right)}
\end{aligned}
$$


corresponde à parte advectiva do problema, sendo $\mathbf{U}=\left(v_{1}, v_{2}\right)$.

Os parâmetros $\lambda_{1}, \lambda_{2} \in[-1,1]$ e seus valores são escolhidos de maneira a assegurar que $\mathcal{B}_{e}(\cdot, \cdot)$ tenha certas propriedades desejáveis, tais como simetria e coercitividade. As funções $\alpha, \beta \geq 0$ são definidas sobre $\Gamma$ por

$$
\left.\alpha\right|_{e}=\alpha_{e}=\frac{\left\{p^{6}\right\}_{e}}{h_{e}^{3}},\left.\quad \beta\right|_{e}=\beta_{e}=\frac{\left\{p^{2}\right\}_{e}}{h_{e}}, \quad \forall e \in \mathcal{E}_{h}
$$

e são chamados de parâmetros de penalização descontínua. A função de estabilização $\gamma$ é definida sobre $\Gamma^{\circ}$ e sua escolha é feita de modo que

$$
\gamma \geq \theta|\mathbf{U} \cdot \nu|
$$

sendo $\theta$ uma constante positiva independente de $e$ e $h$. Observamos que quando a função de estabilização é $\gamma=|\mathbf{U} \cdot \nu| / 2$, a função de fluxo numérico coincide com o fluxo upwind, conforme [1].

Consideremos o funcional linear $l(\cdot)$ sobre $S^{\mathbf{p}}\left(\Omega, \mathcal{T}_{h}, \mathcal{F}\right)$, associado com $f, g_{0}$ e $g_{1}$, definido por

$$
\begin{gathered}
l(\phi)=l_{\Delta}(\phi)+l_{s}(\phi), \\
l_{\Delta}(\phi)=\sum_{\kappa \in \mathcal{T}_{h}}(f, \phi)_{L^{2}(\kappa)}+\lambda_{1}\left\langle g_{0}, \nu \cdot \nabla(\Delta \phi)\right\rangle_{L^{2}(\partial \Omega)}-\lambda_{2}\left\langle g_{1}, \Delta \phi\right\rangle_{L^{2}(\partial \Omega)}, \\
l_{s}(\phi)=\left\langle\alpha g_{0}, \phi\right\rangle_{L^{2}(\partial \Omega)}+\left\langle\beta g_{1}, \nu \cdot \nabla \phi\right\rangle_{L^{2}(\partial \Omega)} .
\end{gathered}
$$

Com base no que apresentamos acima, podemos introduzir, agora, o seguinte método de elementos finitos de Galerkin descontínuo: encontre $\psi_{\mathrm{DG}} \in S^{\mathbf{p}}\left(\Omega, \mathcal{T}_{h}, \mathcal{F}\right)$ tal que,

$$
\mathcal{B}_{\mathrm{DG}}\left(\psi_{\mathrm{DG}}, \phi\right)=l(\phi) \quad \forall \phi \in S^{\mathbf{p}}\left(\Omega, \mathcal{T}_{h}, \mathcal{F}\right) .
$$

De modo a assegurar que (3.2) seja significativo, assumimos que $p_{\kappa} \geq 3$ para todo $\kappa \in \mathcal{T}_{h}$.

\section{Resultados Numéricos}

Para analisar o desempenho do método DGFEM apresentado na seção anterior, vamos aplicá-lo para resolver numericamente o problema da cavidade. Esse problema tem sido amplamente usado na literatura como teste de validação. Aqui vamos resolvê-lo para números de Reynolds 100, 400 e 1000, e vamos comparar o desempenho do DGFEM com os resultados apresentados em [6], que é largamente referenciado na literatura como um importante "benchmark" para esta aplicação.

O domínio do fluxo da cavidade consiste do quadrado $(0,1) \times(0,1)$ e a velocidade do fluido na fronteira superior é $u=1, v=0$, enquanto que, nas demais fronteiras, $u=0, v=0$. Portanto, em termos da função corrente $\psi$, chegamos no seguinte problema de valor de fronteira:

$$
\nu \triangle^{2} \psi-\frac{\partial}{\partial x}\left(\frac{\partial \psi}{\partial y} \triangle \psi\right)+\frac{\partial}{\partial y}\left(\frac{\partial \psi}{\partial x} \triangle \psi\right)=0 \quad \text { em } \Omega=(0,1) \times(0,1),
$$




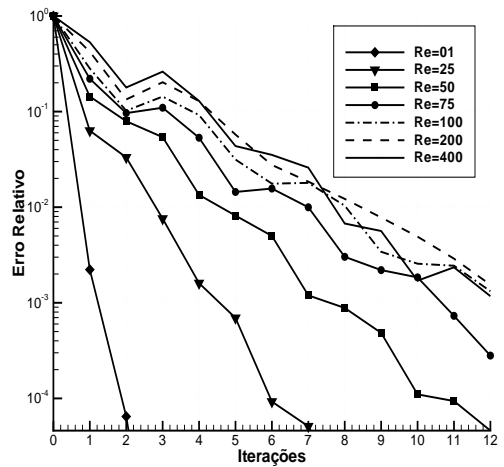

Figura 1: Histórico da convergência.

$$
\begin{array}{rll}
\psi & =0 & \text { sobre } \partial \Omega, \\
\mathbf{n} \cdot \nabla \psi & =0 & \text { sobre } \partial \Omega \backslash \Gamma^{s}, \\
\mathbf{n} \cdot \nabla \psi & =1 & \text { sobre } \Gamma^{s},
\end{array}
$$

sendo $\Gamma^{s}$ a fronteira superior da cavidade.

Para o cálculo da solução numérica, usamos uma malha uniforme composta de $47 \times 47$ elementos quadrados e, para a aproximação polinomial, usamos polinômios descontínuos por parte de grau igual a 3 em cada uma das variáveis.

Para linearizar a equação, conforme mencionado anteriormente, usamos o método de iteração de Picard, desse modo, para $\operatorname{Re}=100$ e $R e=400$, a aproximação inicial de $v_{1}$ e $v_{2}$ é tomada como sendo nula. Já para $\operatorname{Re}=1000, v_{1}$ e $v_{2}$ são tomados como o resultado obtido no cálculo de $R e=700$, onde, por sua vez, foi usado como aproximação inicial o resultado do cálculo de $\mathrm{Re}=400$. Em todos os casos, foi usado como critério de parada um limitante para o erro relativo $e_{r}=\left\|\psi_{i}-\psi_{i-1}\right\| /\left\|\psi_{i}\right\|$ em que $\psi_{i}$ é a solução numérica do DGFEM na iteração $i$.
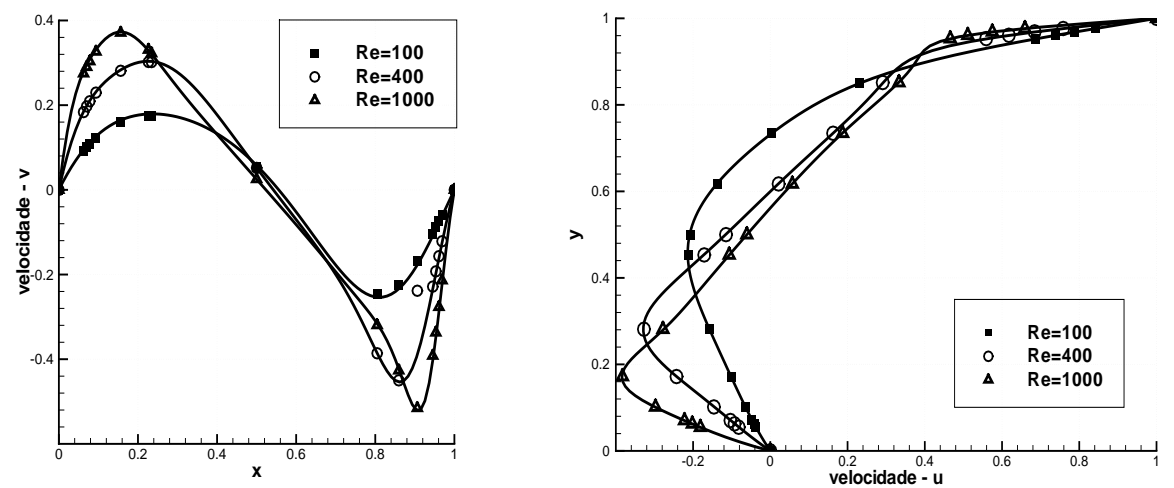

Figura 2: Perfil das velocidades horizontal(esquerda) e vertical(direita) 
Os parâmetros $\lambda_{1}, \lambda_{2}, \theta$, e $\gamma$ da forma $\mathcal{B}_{\mathrm{DG}}(\cdot, \cdot)$ são escolhidos como sendo $-1,-1,0.5$ e $\theta|\mathbf{U} \cdot \nu|$, respectivamente. A escolha dos dois primeiros assegura que a forma bilinear $\mathcal{B}_{e}(\cdot, \cdot)$ é não-simétrica e positiva definida, enquanto que os dois últimos, garantem que o fluxo numérico seja o fluxo upwind.

Na Figura 1 apresentamos o histórico da convergência do erro relativo $e_{r}$ para o problema da cavidade, usando diferentes números de Reynolds. Observamos que para Reynolds 700, foram necessárias 4 iterações além das 12 contabilizadas no cálculo de Reynolds 400. Já para Reynolds 1000, também foram necessárias 4 iterações além das contabilizadas no cálculo de Reynolds 700. Sendo que para ambos os casos, o erro relativo foi menor que 0.0083 .

Um resultado referente ao desempenho do DGFEM é apresentado na Figura 2, onde comparamos o perfil da velocidade horizontal e vertical ao longo do centro da cavidade, com os resultados obtidos por [6] para números de Reynolds 100, 400 e 1000. Nestes gráficos, as linhas sólidas representam a solução numérica do DGFEM, e os símbolos representam os valores apresentados em [6]. Pode-se observar aqui uma ótima concordância entre estes resultados, comprovando que o método é eficiente.

Uma seqüência de imagens das linhas de contorno do número de Mach para Reynolds 100, geradas durante o processo iterativo, é apresentada nas Figuras 3 - 11. Observa-se que a Figura 3 é simétrica, pois a aproximação inicial tomada para $v_{1} \mathrm{e} v_{2}$ são nulas. Por último, na Figura 12, apresentamos as linhas de contorno do número de Mach obtidas na última iteração para $\operatorname{Re}=400$ (esquerda) e $R e=1000$ (direita).

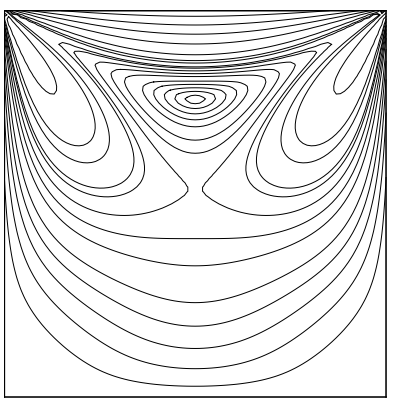

Figura 3: Iteração 00.

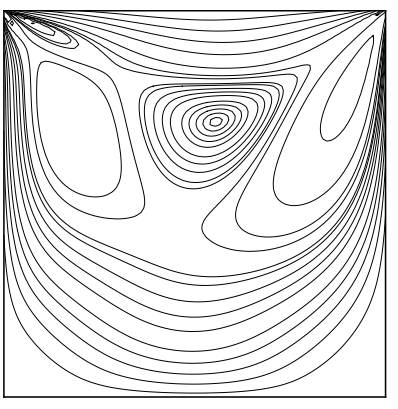

Figura 4: Iteração 01.

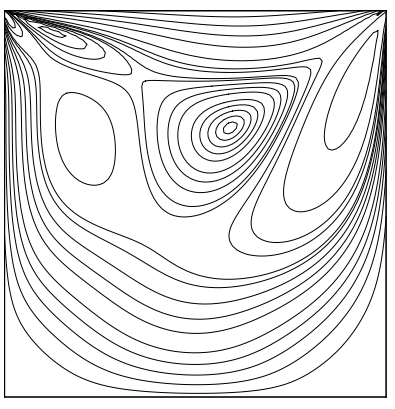

Figura 5: Iteração 02.

\section{Conclusão}

Um método de elementos finitos de Galerkin descontínuo foi desenvolvido para uma equação biharmônica não-linear, associada com a formulação de função corrente para as equações de Navier-Stokes incompressíveis. O método apresentado é uma combinação do DGFEM não simétrico para a equação biharmônica e de uma versão estabilizada do DGFEM para problemas hiperbólicos. Este método tem alta ordem de precisão, é localmente conservativo e admite variação da ordem de aproximação de um elemento para outro com extrema facilidade. Uma série de experiências 


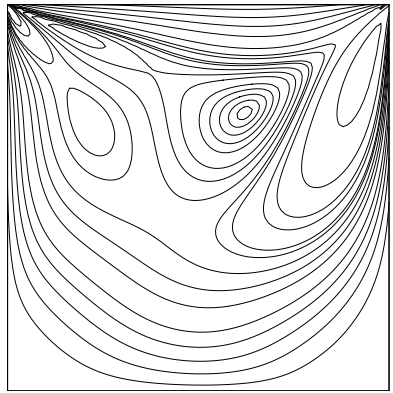

Figura 6: Iteração 03.

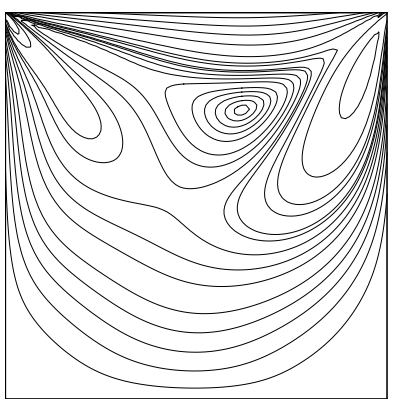

Figura 9: Iteração 07.

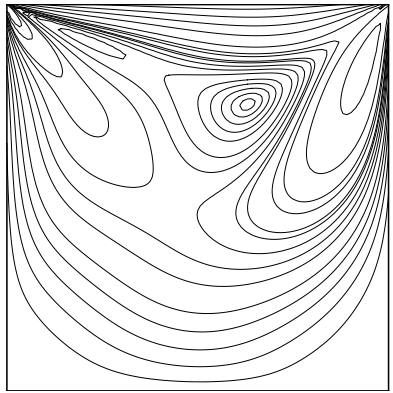

Figura 7: Iteração 04.

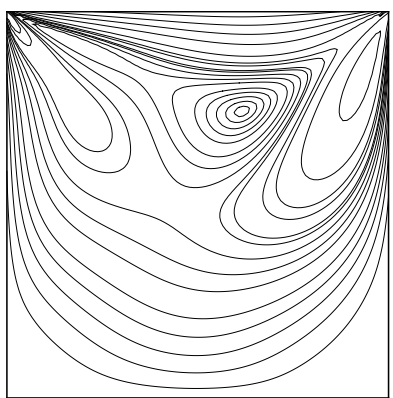

Figura 10: Iteração 09.

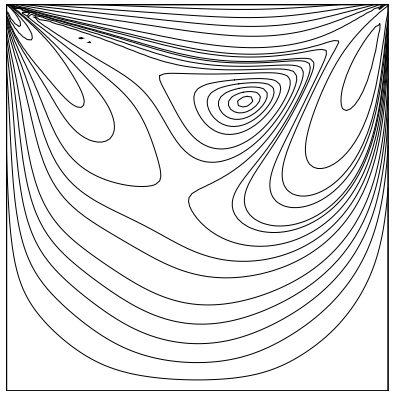

Figura 8: Iteração 05.

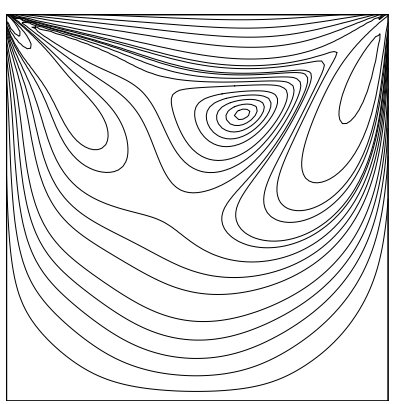

Figura 11: Iteração 12.
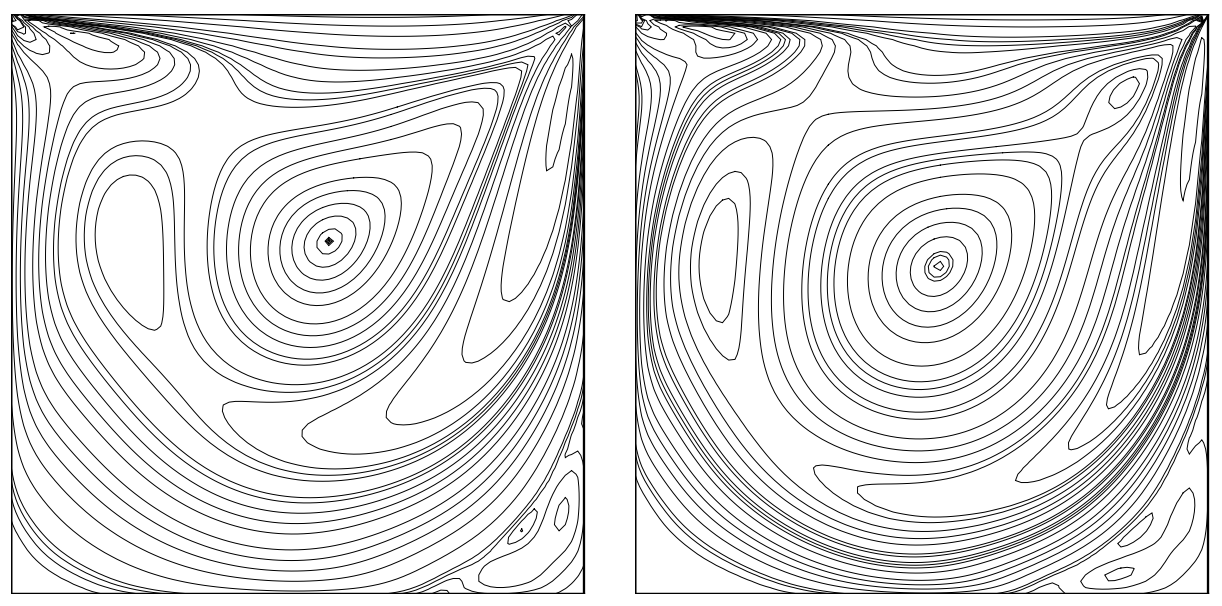

Figura 12: Linhas de contorno do número de Mach para Re $=400$ (esquerda) e $\mathrm{Re}=1000$ (direita).

numéricas realizadas para o problema de teste do fluxo em uma cavidade bidimensional, comprovou a eficiência do método para ampla escala do número de Reynolds. 
Abstract. In this paper we consider discontinuous Galerkin methods for 2D steady state incompressible Navier-Stokes equations. Using a stream function formulation, the problem is reduced to a biharmonic non-linear equation which is linearized by the Picard iteration method. For resulting linear biharmonic equation we introduce a discontinuous Galerkin finite element method with interior penalties. The method is resulted from combination other two formulations of discontinuous Galerkin methods for elliptic part and hyperbolic part of the equation. The numerical results comproved the efficiency of the method for a wide range of the Reynolds number.

\section{Referências}

[1] F. Brezzi, L. Marini e E. Süli, "Discontinuous Galerkin methods for first-order hyperbolic problems", Technical Report 04/02, Oxford University Computing Laboratory, 2004.

[2] B. Cockburn, G. Kanschat, D. Schötzau e C. Schwab, Local discontinuous Galerkin methods for the Stokes system, SIAM J. Numer. Anal., 40, No. 1 (2002), 319-343.

[3] B. Cockburn, G. Kanschat e D. Schötzau, The local discontinuous Galerkin method for linear incompressible fluid flow: A review, Computer and Fluids, 2003. published online.

[4] B. Cockburn, G. Kanschat e D. Schötzau, A locally conservative LDG method for the incompressible Navier-Stokes equations, Math. Comput., 2005. online.

[5] B. Cockburn, G. Kanschat e D. Schötzau, Local discontinuous Galerkin methods for the Oseen equations, Math. Comput., 73 (2004), 569-593.

[6] U. Ghia, K.N. Ghia, e C.T. Shin, High-Re solutions for incompressible flow using the Navier-Stokes equation and a multigrid method, J. comput. Phys., 48 (1982), 387-411.

[7] V. Girault e P. Raviart, "Finite Element Methods for Navier-Stokes Equations, Theory and Algorithms", Spring-Verlag, Berlin, 1986.

[8] H.P. Langtangen, K. Mardal e R. Winther, Numerical methods for incompressible viscous flow, Advances in Water Resources, 25 (2002), 1125-1146.

[9] I. Mozolevski e E. Süli, A priori error analysis for the hp-version of the discontinuous Galerkin finite element method for the biharmonic equation, Comput. Meth. Appl. Math., 3, No. 4 (2003) 596-607.

[10] R. Rannacher, Finite element methods for the incompressible Navier-Sstokes equations, http://www.iwr.uni-heidelberg.de/sfb359/Preprints1999.html 1999.

[11] E. Süli e I. Mozolevski, hp-version interior penalty DGFEMs for the biharmonic equation, Technical Report 04/05, Oxford University Computing Laboratory, 2004. 
[12] M.F. Wheeler, An elliptic collocation finite element method with interior penalties, SIAM J. Numer. Anal., 15 (1978), 152-161. 